\title{
SUBMICRONIC PATTERNS USING LITHOGRAPHIC TECHNOLOGIES FOR FUTURE APPLICATIONS IN THE FIELD OF SENSORS
}

\author{
Liliana-Laura Badita ${ }^{1}$, Aurel Zapciu², Valentin Gornoava ${ }^{3}$ \\ 1,2,3 National Institute of Research and Development for Mechatronics and Measurement Technique, \\ Bucharest, Romania \\ badita1@yahoo.com,zapciu.aurel@yahoo.com, valentin_gornoava@yahoo.com
}

\begin{abstract}
In this article are presented the main results of a project realized at INCDMTM whose main objective was to obtain submicronic patterns that can be used later for the design and manufacture of microsensors. Predefined submicronic geometric patterns were performed using electron beam lithography and atomic force microscopy lithography on silicon and copper substrates. Predefined geometric shapes could be fully accomplished using electron beam lithography only on silicon samples. These patterns could not be obtained on the copper layer, probably due to the high roughness of the metallic layer. Using atomic force microscopy lithography, simple submicron patterns were made, even very simple compared to the predefined ones, on both types of substrate. Also, based on the atomic force microscopy analysis and the determination of the tribological parameters of the surfaces it was observed that nano-scale depth patterns were obtained using both types of lithography. Thus, it was concluded that predefined geometric submicronic patterns were realized completely and precisely only on the silicon substrate using electron beam lithography. Through an experimental development, it will be possible to make submicronic patterns as complete as possible which in the future can be used for the manufacture of sensors. These types of sensors will be implemented in complex mechatronic systems.
\end{abstract}

Keywords: Lithography, Electron Beam Lithography, Submicronic Patterns, Sensors, Atomic Force Microscopy.

\section{Introduction}

Obtaining submicronic patterns that could be used to design and manufacture microsensors was the main objective of a project, realized at INCDMTM, whose results can be of great importance in the development of cyber-mechatronic systems.

The use of microsensors comprises the majority of industrial environments (machines manufacturing, fine mechanics, mechatronics, electronics, electrotechnics, aerospace industry, iron and steel industry, metallurgy, etc.), economics, information society, etc. In industry, they are mainly used for the automation of industrial processes. [1], [2]

Sensor is a device that transforms a variation of a mechanical or other nature size into a variation of electrical size. Sensors can measure variations of different mechanical sizes: forces, torques, pressures, linear or angular displacements, speeds, accelerations, etc. (Figure 1). [3], [4]

The sensor provides an electrical signal proportional to the intensity of the measure of which variation is monitored. The automatic regulating of a process or the protection of an installation is made by decisions taken by a program that analyzes the signals received from it.

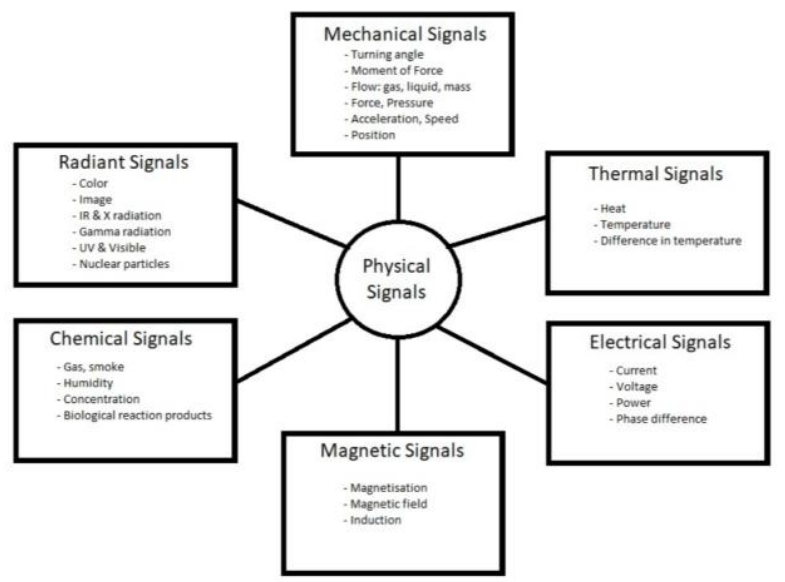

Figure 1: Types of sizes that can be transformed by transducers into measurable physical signals [3]

Generally, a sensor or a sensors assembly comprises the transducer/ transducers for converting the input to a useful electrical signal, which can be processed by measuring instruments or computers, as well as circuits for adapting and 
converting signals and, eventually, for processing and evaluating information.

The main characteristics of the sensors can be defined by the following parameters: field of use; resolution (sensitivity - lowest measurable stimulus increment); selectivity - the maximum frequency of the detectable stimulus; accuracy - reported measurement error in percent, at full scale; dimensions and mass of the sensor; operating temperature and environmental conditions; lifetime (in hours or number of operating cycles); long-term stability and the cost.

The range of microsensors comprises structural and functional groups of sensors and microsensors in different constructive variants based on different operating principles (inductive, capacitive, magnetic, photoelectric, piezoelectric, ultrasonic, interferometric, telemetric, etc.) along with the transducers and micro transducers groups found in many mechanical/ micromechanical, mechatronic/ micro mechatronic constructive variants.

Sensors are classified according to several criteria. In Table 1 are shown the main types of sensors classified according to the signals they measure. [5]

Table 1. Classification of sensors according to the signals they measure

\begin{tabular}{|c|c|}
\hline Signal's shape & Measured sizes \\
\hline Thermal & $\begin{array}{l}\text { Temperature, heat, heat flow, } \\
\text { entropy, heat capacity, etc. }\end{array}$ \\
\hline Radiant & $\begin{array}{l}\text { Gamma rays, x-rays, ultraviolet, } \\
\text { visible and infrared light, } \\
\text { microwaves, radio waves, etc. }\end{array}$ \\
\hline Mechanical & $\begin{array}{l}\text { Displacement, speed, acceleration, } \\
\text { force. Pressure, mass flow, } \\
\text { amplitude and acoustic } \\
\text { wavelength, etc. }\end{array}$ \\
\hline Magn & $\begin{array}{l}\text { Magnetic field, flow, magnetic } \\
\text { moment, magnetization, magnetic } \\
\text { permeability, etc. }\end{array}$ \\
\hline Chemical & $\begin{array}{l}\text { Humidity, pH level and ions, gas } \\
\text { concentration, toxic and } \\
\text { flammable materials, vapors and } \\
\text { odors concentration, pollutants, } \\
\text { etc. }\end{array}$ \\
\hline Biological & $\begin{array}{l}\text { Sugars, proteins, } \\
\text { antigens, etc. }\end{array}$ \\
\hline
\end{tabular}

At present, there are sensors for more than 100 physical sizes, and if sensors for different chemicals are also taken into account, their number is hundreds.

Depending on the type of the input physical quantity, sensors can be classified into [6]:

- absolute, when the electrical output signal can represent all possible values of the physical input quantity, relative to a chosen origin (reference);
- incremental, when an origin cannot be determined for all points in the measuring range, but each measured value is the origin for the next one.

Depending on the type of output quantity, there are [6]:

- analogue sensors, for which the output signal is always proportional to the input physical quantity;

- numerical (digital) sensors, for which the output signal can only take a limited number of discrete values, which allow quantification of the physical input signal.

Another classification criterion takes into account the number of transducers and the number of dimensions assigned to the measured values. Thus, the sensors are classified into [6]:

- scalars (a transducer, a dimension);

- vectorial (measurements in three orthogonal directions);

- matrix (a certain number of transducers arranged after a mono-, bi- or threedimensional matrix).

Another sensor classification divides them into:

- passive sensors that directly generate electrical signal in response to external stimuli. This means that the stimulus energy is converted by the sensor into output energy without an additional power source, such as thermocouples, pyroelectric detectors, piezoelectric sensors.

- active sensors that require external power. Active sensors are sometimes referred to as parametric, because the change of intrinsic properties under the action of input quantity induces the change of the output electrical signal. Sensors stimuli can be of physical or chemical nature, such as radiations, temperature, pressure, vibration, speed, ions concentration, etc.

First developments of micro and nanosensors have been valorized on a mature infrastructure made of Si built for the integrated circuit industry (IC), and recent developments have led to the use of materials and processes not associated with IC manufacturing. The main materials used today are: carbon nanotubes; nanowires and nanocrystals; porphyrins; conductive polymers (e.g., polyaniline (PANi) and its derivatives, polydiphenylamine, polypyrrole (PPy) and its derivatives, polythiophene (PTh) and its derivatives, polyfenazine polyacrydine; polytiazine; polyfluorene, polyluminol, polyrodamines, polytryphenylamines); graphene; silicon based materials (e.g. monocrystalline silicon, polycrystalline and amorphous silicon, porous silicon, silicon dioxide, silicon nitride); germaniumbased materials (e.g. polycrystalline germanium, polycrystalline SiGe); metals (e.g. aluminum, gold, chrome, titanium); severe semiconductors (e.g. silicon carbide, diamond, gallium arsenic); 
piezoelectric materials (e.g. lead zirconium titanate). [7]

Microsensors are manufactured using various manufacturing techniques that involve the creation of nanostructures by different technologies, including [8]:

- Lithography;

- Silicon processing in volume - by anisotropic corrosion;

- Microprocessing on the surface of silicon - by CVD deposition, physical methods deposition, dry and wet corrosion, impurification, implantation, diffusion, sacrifice corrosion;

- LIGA technique, which involves the reunion of three technologies: lithography, galvanic deposition of layers and formation;

- SOI manufacturing techniques by implantation or deposition.

Lithography consists of transferring a pattern from a template (physical or virtual mask) to a substrate made of various materials (metal, nitride, oxide, silicon, glass, GaAs, etc.). This pattern is then used to engrave a thin base film (such as an oxide or a nitrate) for different purposes. [9]

The main types of lithography are: photolithography, optical lithography, electron beam/ ion beam lithography, nanoimprint lithography, X-rays lithography, Scanning Probe Lithography, etching. [10]

Photolithography, optical lithography or UV lithography [11], [12] use UV light at different wavelengths in order to transfer a pattern from a mask to a substrate, usually, a photoresist. This technique can easily produce periodical or quasiperiodical nanostructured models on optical fibers, suitable to be used in the manufacture of sensors with optical fiber [13]

The principle of optical lithography consists in the selective removal of some parts from a photosensitive polymer film to transfer a geometrical scheme over the sample using a mask the draw of the scheme is realized by a metallic layer applied to a quartz glass.

A technology developed as alternative to photolithography in terms of cost-effectiveness is UV-nanoimprint lithograpgy (UV-NIL). This method is used to obtain a high resolution $(<10 \mathrm{~nm})$ in lithography. It is a solution for the new generation of semiconductors, MEMS and optoelectronics.

Optical lithography by immersion is another developing variant, in which a thin liquid layer (usually water) is interposed between the optical system and the sample. Because the refractive index of the liquid is higher than that of the environment, it is possible to create even smaller structures on the sample.

Electron beam lithography can define high resolution nanostructured patterns $(\sim 10 \mathrm{~nm})$ and over large areas $\left(>1 \mathrm{~cm}^{2}\right)$. It uses an electron beam accelerated at $5 \mathrm{kV}$ to $100 \mathrm{kV}$ (or even more in some special applications) to expose a substrate coated with electronoresist. [12]

The electron beam allows direct patterning without the need for pre-existing masks. Electronresists are the environment on which irradiation produced by the electron beam and a subsequent development turn it into a mask for transferring a pattern on the working substrate of a configuration. Typically, electron-resists are polymers dissolved in a liquid solvent. This liquid resist is applied to the substrate by "spin coating" at rotative speeds ranging from 500 to $6000 \mathrm{rpm}$. The polymer film thus deposited is heat treated until the solvent is evaporated and the polymer undergoes a phase transition (to the glassy phase).

PMMA (polymethylmethacrylate) was one of the first materials used as electron-resist and is still widely used for this purpose. It shows an excellent resolution but is not very sensitive. [14]

The electron beam that exposes the electronresist is made of electrons accelerated at voltages of kilovolts order. When these electrons enter the sample, they interact with the electric fields of the sample atoms. The electrons in the beam can undergo elastic scatterings (without energy loss) altering their movement direction, and after a certain number of deflections, these electrons can even return backward leaving the sample.

The electrons passing through the PMMA thin layer reach the silicon and a fraction of $18 \%$ are retro-scattered (backscattered). They cross again through the PMMA layer, this time in the opposite direction. For PMMA deposited on silicon and irradiated with an electron beam with $20 \mathrm{keV}$ energy, about $5 \%$ of the beam energy is dissipated in PMMA by the electrons in the primary beam, $4 \%$ is dissipated in the same layer by the backscattered electrons, $85 \%$ thermic dissipates in the substrate, and $6 \%$ is the energy of the backscattered electrons leaving the sample.

The energy loss of the electrons beams in the electron-resist layer leads to the emission of secondary electrons (through inelastic crash processes), and these electrons are the ones that break the molecular chains of the polymer (electronresist) converting it into a fraction with smaller molecular mass. This fraction is dissolved in a developer, leaving only the non-irradiated electronresist areas on the substrate.

The typical PMMA developer is metaisobutylketone - MIBK. The undiluted MIBK is, however, too energetic and it also removes unexposed areas of resist. Therefore, it is used dilute in isopropyl alcohol (IPA) in a 1:3 ratio (three parts IPA and one MIBK) to obtain a very large contrast.

Compared to photolithography, electron beam lithography has the following advantages:

- precise control of the released energy on the substrate coated with resist; 
- deviation and modulation of the electron beam with velocity and precision through the magnetic or electrostatic field;

- focusing the electrons beam in a point of dimensions $<100 \AA$, compared to the spot made by the light beam, which is of $5000 \AA$

- realization of physical mask is not necessary (software mask is enough);

- precision rendering ability on small surfaces of the substrate;

- low defects density;

- large depth at one point due to continuous focusing on various geometries;

and disadvantages:

- rapid scattering of electrons in the solid practically limits the resolution to $10 \mathrm{~nm}$;

- electrons, charged particles must be kept in vacuum, requiring a more complex equipment than photolithography;

- due to the small exposure speed, the electrons beam must scan the whole structure, requiring a high exposure time depending on the necessary geometry, spot's diameter, resolution and resist's sensitivity.

Printing the structure on the electron-resist can be done in the following ways:

- an opaque mask at electrons is made on electron-resist, the large electron beam is projected onto the electron-resist through this mask;

- the structure of a photoemitter is designed and imprinted on electron-resist;

- a very small diameter electron beam is scrolled on the surface of the electron-resist, being obturated or unobturated according to the printed structure.

The equipment that can perform this type of lithography is divided into two main categories:

- Scanning electron microscopes converted to lithography

- Equipment dedicated to electron beam lithography

Electronic microscopes and some of the dedicated equipment use beams with Gaussian profile of the current's intensity, for writing. Dedicated equipment, with applications for photolithographic masks realization, uses to expose electron beams with predefined geometry.

In most cases these beams have square or rectangular section. Sectional dimensions of these beams can reach tens of $\mu \mathrm{m}$. On the other hand, the minimum beams size is of the hundreds of nm order and as such, and the resolution of this type of machine is lower than the resolution of Gaussian beam machines.

Focused ion beam lithography involves the use of ions instead of electrons in the photolithography process. This has two great advantages: due to the much larger mass, the ions are much less scattered in resist than electrons, and the secondary electrons produced by ion bombardment have low energies.

For these reasons, proximity effects are lacking, a resolution higher than the one with electron beam can be achieved, and the dose is $1-10 \%$ of the exposure dose with electron beam of the resist. The Broglie wavelength equivalent is in the range 0.28 $0.9 \mathrm{pm}$, depending on the chosen element and the state of the load, such as, for example, for the $\mathrm{H}^{+}$ protons accelerated to $10-100 \mathrm{keV}$. Diffraction effects in the mask are missing. Due to the high focusing depth, it can be used for the lithography of topographies with large-aspect factor such as the manufacture of TFT (Thin Film Transistor) displays. Resists' sensitivity (PMMA) is larger with orders of magnitude towards ions than electrons. Ion beams can be used in both projection exposure systems and direct exposure systems, without mask. The ions sources may be from gaseous field, liquid metal or microplasma. [12]

Projection exposure systems use radiofrequency sources for the production of ions $\left(\mathrm{H}^{+}, \mathrm{He}^{+}\right.$or $\left.\mathrm{Ar}^{+}\right)$in the range of $10-100 \mathrm{keV}$, managing to expose fields of $5 \times 5 \mathrm{~mm}$ order. FIB focused ion beam systems can be used for direct implantation on the plate for contamination, without using masks or resists. This technique eliminates many sources of contamination.

X-ray lithography is a technique for transposing, keeping the resolution of a structure from a primary mask onto a resist $X$ that is in contact with the substrate (Figure 2). [12]

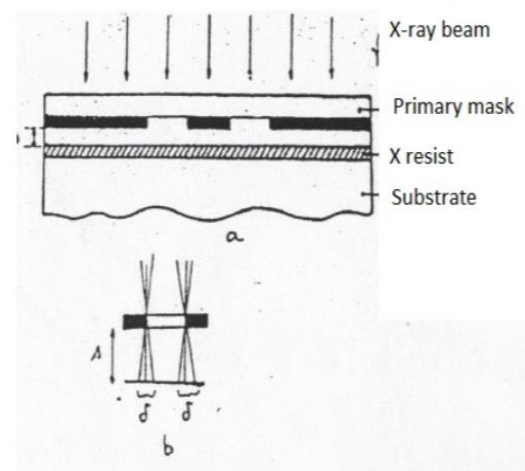

Figure 2: X-ray lithography

The use for copying of X-rays with very low wavelength, $10 \AA$, makes it possible to copy without distortions the structure of the primary mask. The mask has to provide at least a 3/1 contrast under conditions of a minimal X-rays attenuation.

As $\mathrm{X}$ resist, an electron-resist is also used because the polymer molecule absorbing the X-ray emits photoelectrons with random directions that interact with the resist modifying its properties. Compared to electron beam and ion beam lithography, X-ray lithography does not require the use of vacuum and ensures a higher resolution processing. 
There are three types of sources for X-ray lithography, namely: electron bombardment, plasma with laser and synchrotron.

Resolution of X-ray lithography is limited by three factors:

- the penumbra effect is due to the fact that the X-rays cannot be effectively collimated;

- distance of photoelectrons penetration that depends on the photoelectrons energy, i.e. the energy of incident $\mathrm{X}$ radiation;

- the absorption thickness influences the resolution in that the minimum width of the segment that can be reproduced is correlated with the absorbtion thickness in the metallic film of the primary mask.

$\mathrm{X}$-ray lithography is a technique recommended for the industrial copying of the primary masks, requiring relatively simple installations at low cost.

Scanning Probe Lithography is a technique in which microscopic systems are used. Scanning probe microscopy systems involve controlling the movement of an atomic sharp tip near or in contact with a subnanometric precision surface.

Some scanning probe systems use a combination of atomic force microscopy (AFM) and scanning tunneling microscopy (STM) modes: the tip is mounted in a cantilever with electrical connection so that the surface force and the tunnelling current are controlled or monitored. STM systems can be functional in ultra-high cavities (UHV STM) or in air, while AFM systems are functional in the air.

Various systems have been used for this lithographic technique, including STM with constant current, non-contact AFM and AFM with constant tip - protecting layer force, and constant current. [10]

Atomic force microscopy (AFM) ensures a way of observing conductive and nonconductive surfaces. An Atomic Force Microscope is a microscope with a proven sub-nanometric resolution in which the image is acquired by recording the vertical deflection of a cantilever using a laser and a photosensitive diode. The tip attached to this cantilever that is in contact with the sample has a radius below $10 \mathrm{~nm}$ (Figure 3). Once the tip is brought into contact with the sample, it is moved in plan using a piezo-scanner, and the cantilever's deflection is recorded line by line, assembled in a matrix and interpreted in real time with the help of the computer, thus realizing the image of the surface.[10]

Force lithography and electric lithography are important within atomic force microscopy (AFM) lithography.

Force lithography is based on the direct mechanical impact produced by a thin measuring tip on the surface of the sample. Pressure of the tip of the measuring head is sufficient to cause plastic deformation on the surface of the substrate. This type of change has a range of applications in nanoelectronics, nanotechnology, materials science, and so on. It allows the manufacture of electronic components with active nanoscale areas, the recording of super dense information and the study of the materials' mechanical properties. For force lithography, the hardness of the measuring head material exceeds that of the sample. Low roughness of the sample (generally, below $1 \div 10 \mathrm{~nm}$ ) is required. Furthermore, the sample surface must be clean. Force lithography is best carried out on certain polymers, including polycarbonate and polyethylene. Measuring heads with a hardness of up to $5.5 \mathrm{~N} / \mathrm{m}$ are typically used in force lithography.

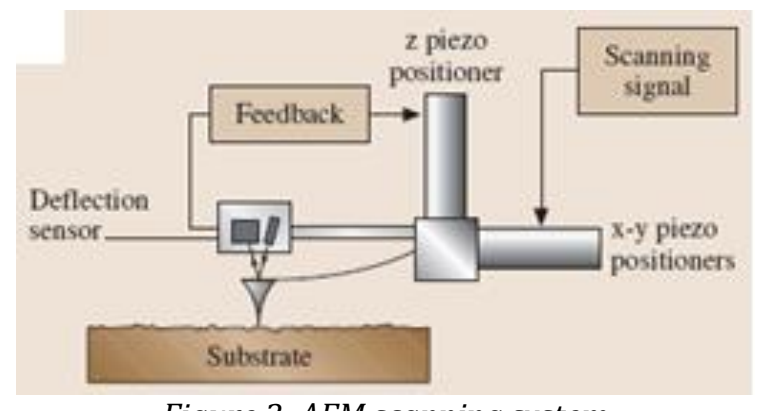

Figure 3: AFM scanning system

Electrical lithography is based on the effect produced by the electric field in the contact area between the tip of the measuring head and the sample surface. When a potential is applied it generates the flowing of an electrons current between the thin measuring head and the sample. The electrical impact may be sufficient to cause local surface change. Modification mechanisms may differ.

It may be the local thermal impact due to high current density. In suitable gaseous media decomposition of gas may occur with precipitation of decomposition products. Also, the evaporation in electric field of the sample material or vice versa of the measuring head material can take place.

A large group of surface modification methods refers to chemically induced chemical transformations in the near-region, particularly, oxidation. The result is the creation of a quantity of oxide below tip, modifying the electronic properties of the sample. The oxidation processes are electrolytic. They are associated with the presence of a water layer adsorbed on the surface of the metallic film. These phenomena are of practical interest due to possible applications to the formation of nanoelectronic elements and layers.

The sample must be a conductor or a semiconductor. A substrate with conductive contact will be used to mount the sample in order to allow the tension between the measurement head and the sample to be applied.

For electric lithography, measuring heads with conductive coatings are used. Typically, semicontact measuring heads with TiN coatings, $\mathrm{W}_{2} \mathrm{C}$ and diamond-like coating are used.

When electric lithography is performed, there are two major options for changing the sample surface: 
changing sample's topography or changing the physical properties of the sample surface without altering the topography.

Scanning Tunnelling Microscopy (STM) Lithography involves the use of scanning tunneling microscopic systems. In these systems, a polarized voltage is applied to the sample and the tip is positioned sufficiently close to the surface for a tunneling current developed across the hole (Figure 4). [10]

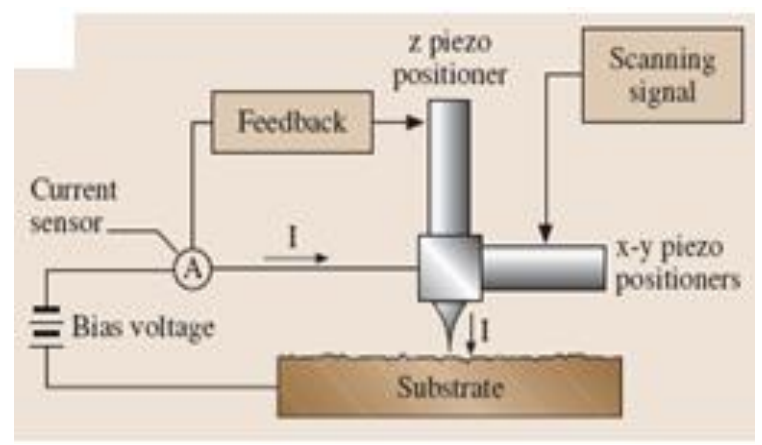

Figure 4: STM system for sample scanning

Because this current is sensitive to the distance between the tip and the surface, tip scanning in the $\mathrm{x}-\mathrm{y}$ plane during the tunneling current recording allows the plotting of surface topography with an atomic scale resolution.

The STM system works only for the conductive surfaces for the need to set a tunneling current.

Electric fields strong enough to induce the emission of the atoms from the tip can easily be generated by applying voltage pulses greater than $3 \mathrm{~V}$. This phenomenon has been used to transfer material from the tip to the surface and vice versa. Ten to twenty nanometric heaps of metals such as $\mathrm{Au}, \mathrm{Ag}$ or Pt have been deposited or moved from a surface in this manner. The same path was used to extract single atoms from the surface of a semiconductor and redeposit it elsewhere. Manipulation of nanoparticles, molecules and single atoms on a surface was also accomplished by simply pushing or sliding them with the STM tip.

Local electrochemical etching and electrodeposition are also possible using STM systems. One drop of the right solution is placed on the substrate. Then the STM tip is immersed in the drop and a voltage is applied. To reduce the faradic currents, the tip is covered with wax so that only the tip is exposed to the solution. Characteristic quantities below $100 \mathrm{~nm}$ were obtained with this technique.

Using a single tip to mass-produce the desired changes in an area leads to very slow manufacturing processes that are not practical for mass production.
With the development of wider surfaces, with individual advances in force, the control of vertical position and of the current, many of these techniques can be used in industrial standard manufacturing processes.

\section{Experimental Methods}

Submicronic patterns that can be used to manufacture integrated sensors in cybermechatronic systems have been developed within this project using two different techniques. It is about electron beam lithography and AFM lithography.

Lithography was chosen to be applied because this technique is a crucial process in producing sensors, integrated circuits, etc.

The main advantages of AFM lithography application are: accuracy of alignment, clear definition of undisturbed area and absence of additional processing stages, such as substrate etching. AFM lithography has the advantage of avoiding damage of the fragile structures, has the potential to greatly expand the ability to model and control the molecular micro-environments.

Of all AFM lithography categories, force lithography was used to achieve submicronic patterns. An NTEGRA Probe NanoLaboratory NTMDT Microscope was used to perform AFM force lithography.

In order to achieve submicronic patterns using electron beam lithography, an electron nanolithography installation was used, consisting of a scanning electron microscope Zeiss Merlin Compact, having a laser interferometry nanolithography system with a resolution of up to 2 $\mathrm{nm}$, from Raith.

Using the two types of lithographic techniques, pattern models were printed on silicon and copper substrates (Figure 5). The copper substrate is a thin film with a thickness of $35 \mu \mathrm{m}$.

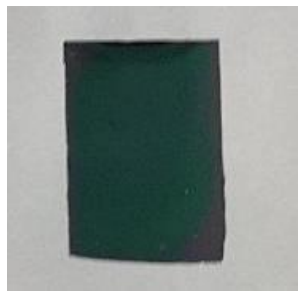

(a)

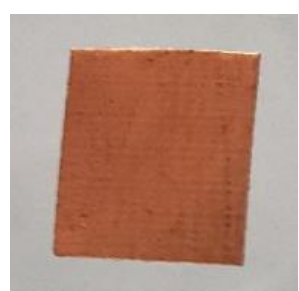

(b)
Figure 5: Plates of Si (a) and plates coated with $\mathrm{Cu}$ thin films (b) with dimensions of $7 \times 10 \mathrm{~mm}$ on which micrometric patterns were realized

Submicronic patterns realized were four predefined geometric shapes (Figure 6) designed with the SolidWorks program. 


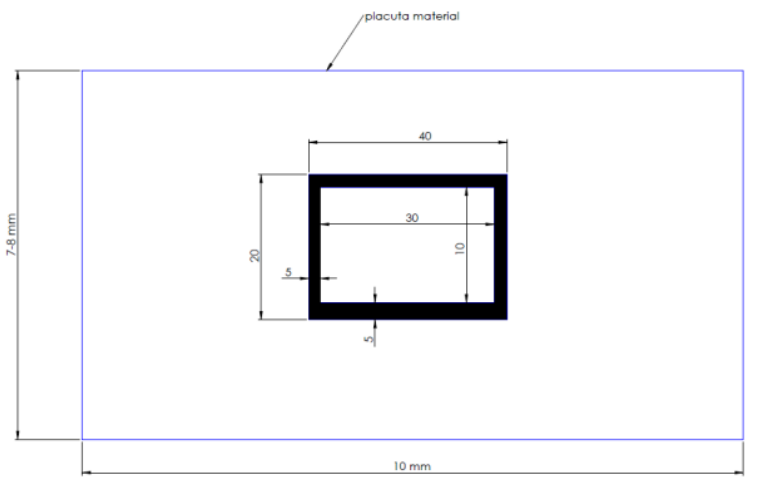

(a)

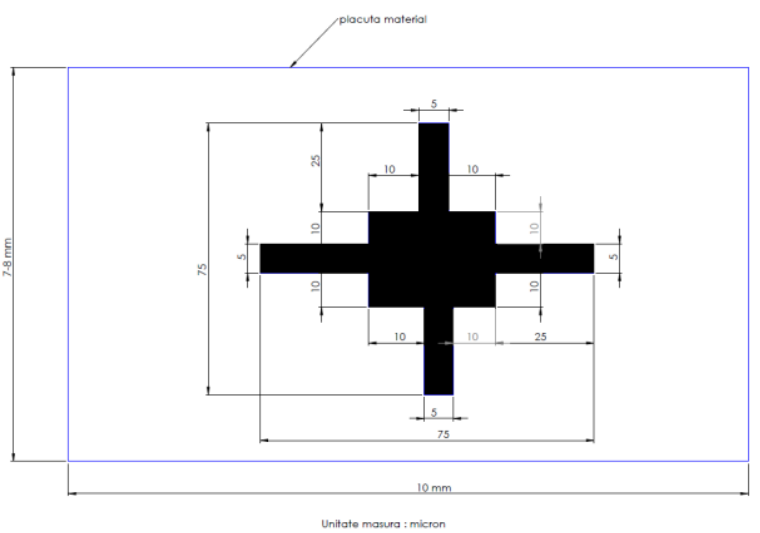

(c)

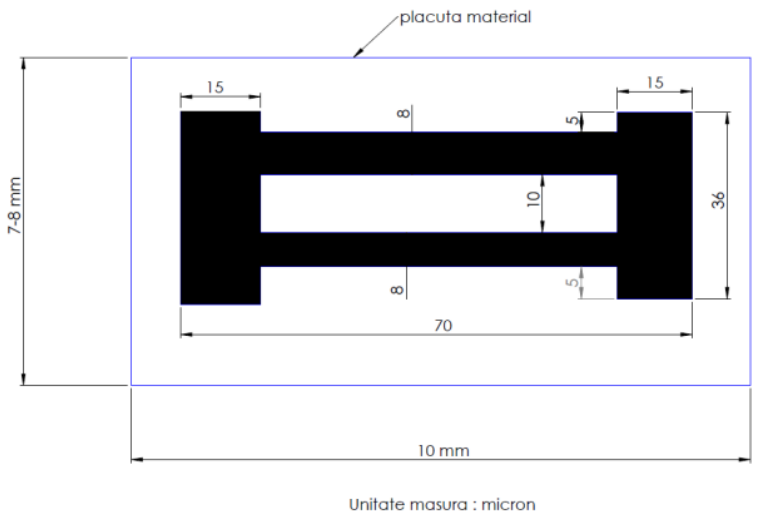

(b)

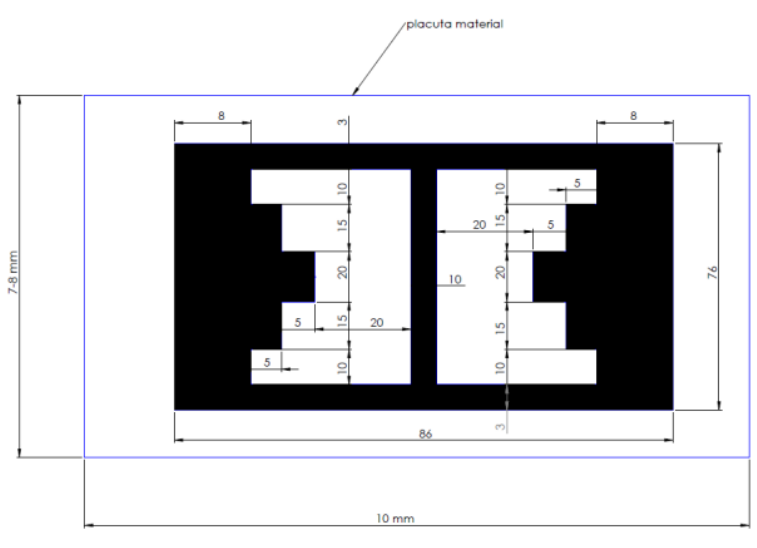

(d)

Figure 6: Predefined geometric shapes designed to make patterns using lithography techniques

The resulted samples were analyzed by atomic force microscopy using the NTEGRA Probe NanoLaboratory NT-MDT microscope.

\section{Results}

On the $\mathrm{Si}$ substrate, using the electron beam lithography, all four predefined geometric shapes were printed for realizing the micrometric patterns (Figure 7).

All these submicronic patterns were analyzed after finalization and it was observed that, in the case of Si samples processed by electron beam lithography, the dimensions of the patterns obtained were very close to those of the projected forms. There is a small difference, which could be considered as error, taking into account that this type of lithography allows realization of the experiments with a high precision.

Predefined geometric shapes could be completely accomplished using electron beam lithography only on Si samples. These patterns could not be obtained on the $\mathrm{Cu}$ layer, probably due to the high roughness of the layer. Its processing prior to the electron beam lithography tests would have destroyed the continuity of the layer.
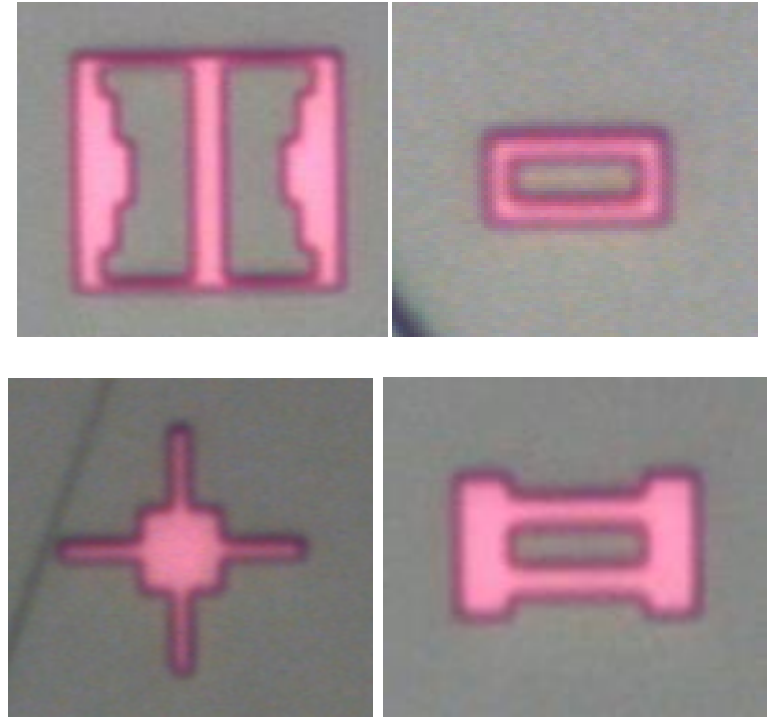

Figure 7: Micrometric patterns printed on Si substrate using electron beam lithography

Using AFM lithography, simpler patterns (Figure 8) have been achieved, less successful in the present case, although AFM lithography has advantages in terms of precision. 


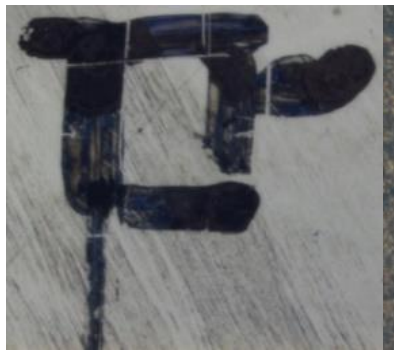

(a)

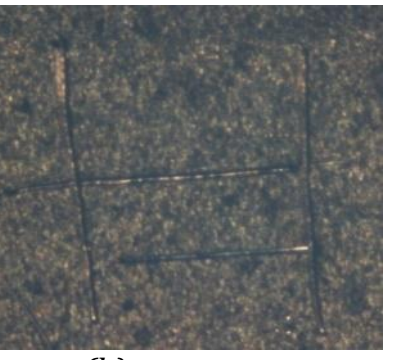

(b)
Figure 8: Micrometric pattern printed on Si substrate

(a) and on Cu thin film substrate using AFM lithography

The resulting samples were analyzed using atomic force microscopy. Part of the analysis results of the samples obtained by electron beam lithography on $\mathrm{Si}$ substrate are presented in Table 2. The 3D patterns and the analysis of the medium profiles on the two axes ( $\mathrm{x}$ and $\mathrm{y}$ ) demonstrate that this lithography method allowed realization of shapes geometrically accurate on the Si substrate.

Using AFM lithography, simple submicronic patterns were made, even very simple compared to predefined ones, on both substrate types - Si and thin $\mathrm{Cu}$ layer (Table 3). Although AFM lithography has advantages such as the precision of lithography, it is possible that in this case the contact force between the measuring head and the surface may not have been large enough to allow complete and powerful scratching of the substrate. Therefore, the results obtained by this technique are not as clear as those obtained by electron beam lithography.

Table 2. AFM analysis of submicronic patterns produced by electron beam lithography on Si substrate

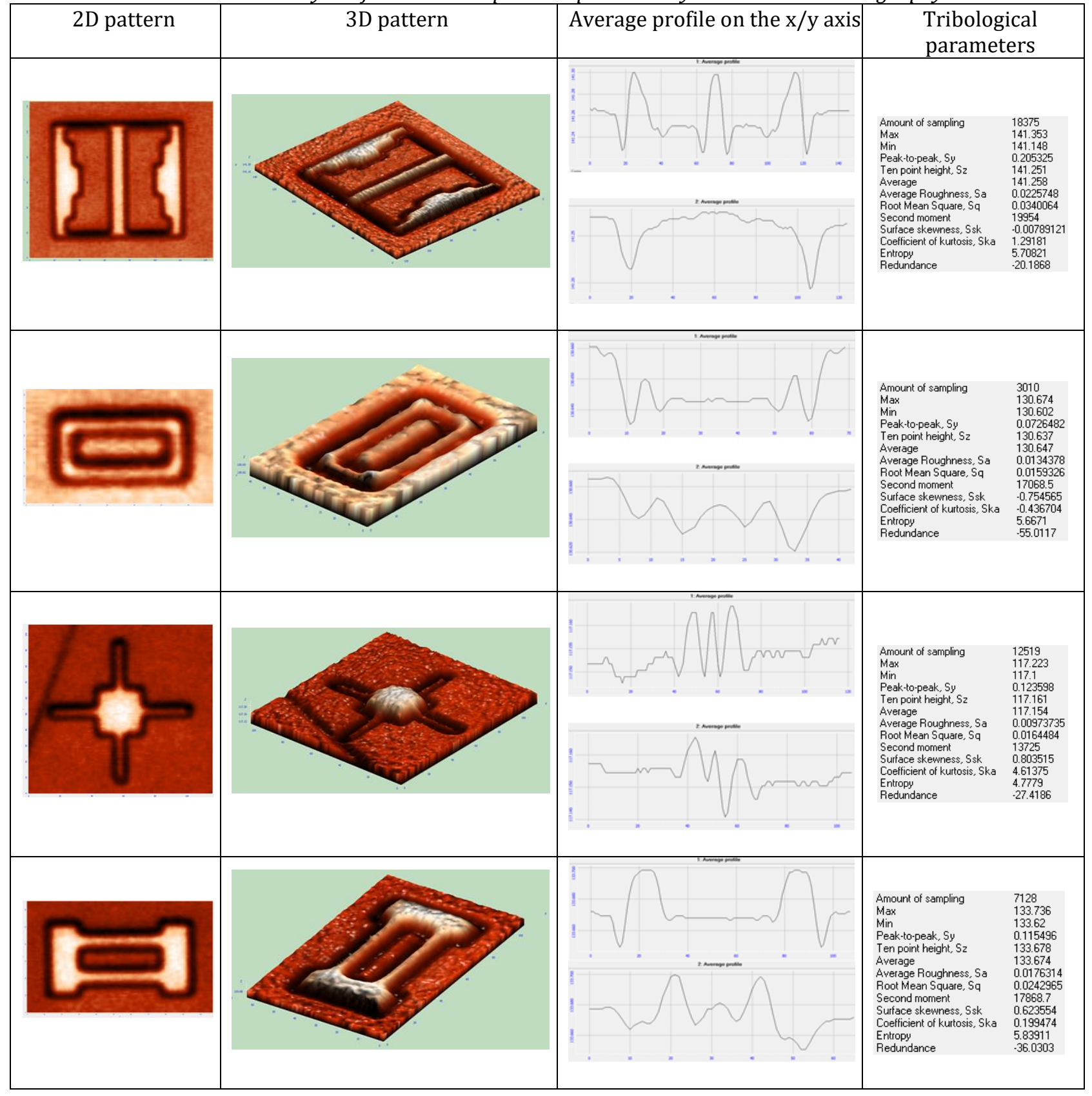


Table 3. AFM analysis of submicronic patterns produced by AFM lithography on Si substrate and thin Cu film.

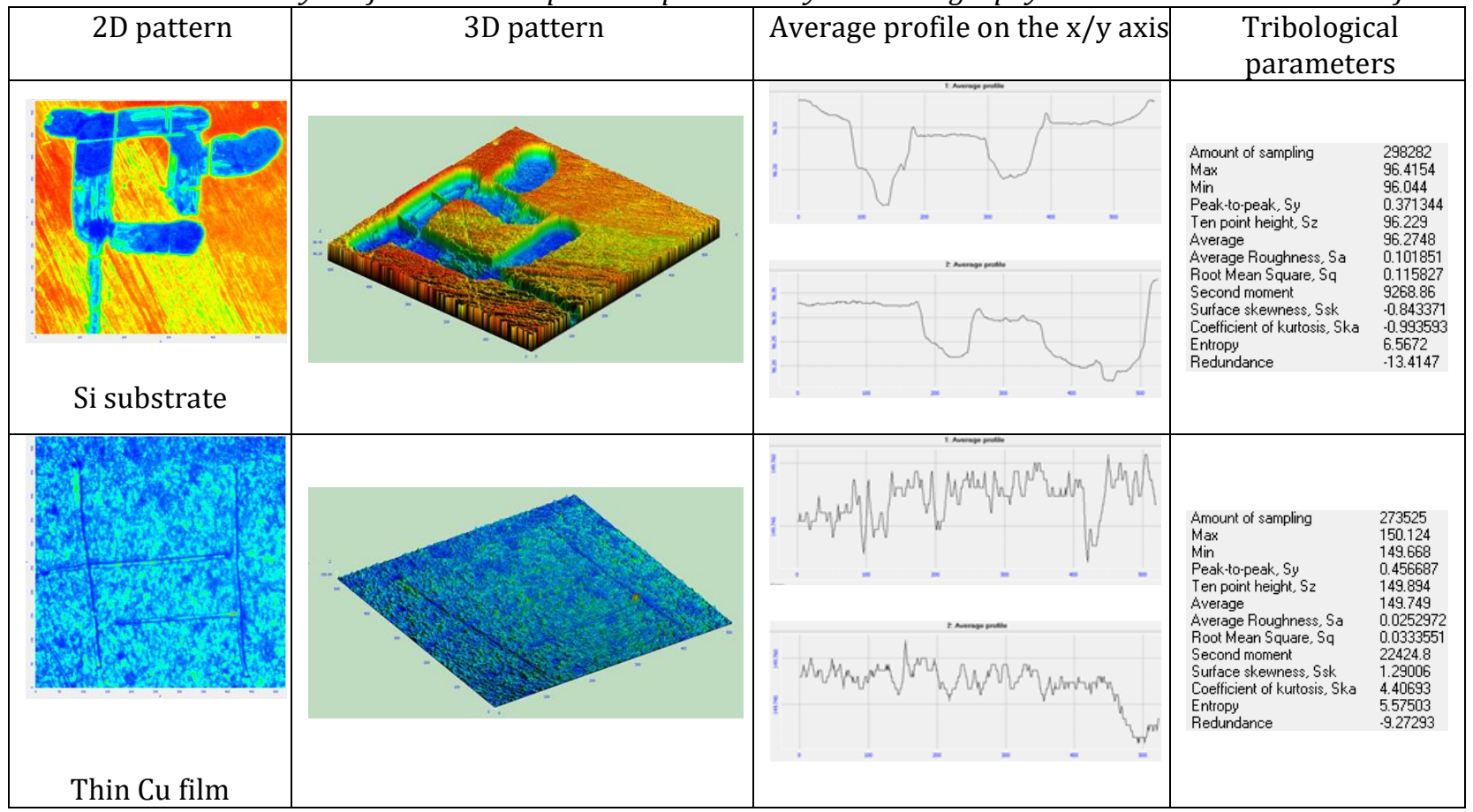

Depth of the patterns obtained by lithography was also determined based on the atomic force microscopy analysis and the determination of the tribological parameters of the surfaces. The average depth values obtained for all types of submicronic patterns made are of the nanometer order, as follows:

- 130,746 nm depth for the patterns obtained on Si substrate by electron beam lithography;

- 96,044 nm depth for patterns obtained on Si substrate by AFM lithography;

- 161,131 nm depth for patterns obtained on thin Cu film by AFM lithography.

The depth of the patterns is higher for the thin $\mathrm{Cu}$ layer than for the Si patterns. One of the reasons could be the hardness of the two materials and the measurement heads of the atomic force microscope used.

\section{Conclusions}

Experiments have demonstrated the possibility of realizing submicronic patterns, although only one of the lithography techniques (electron beam lithography) allowed to obtain superior qualitative patterns.

Analysis of these patterns using atomic force microscopy showed that electron beam lithography allowed realization of precise geometric shapes on the $\mathrm{Si}$ substrate. In the case of samples on $\mathrm{Cu}$ thin layer, using the electron beam lithography, the projected forms could not be obtained.

Also based on the atomic force microscopy analysis and the determination of the tribological parameters of the surfaces it was concluded that nano-scale depth patterns were obtained using both types of lithography.

Thus, the main conclusion is that the predefined geometric shapes could be accomplished completely and precisely only using electron beam lithography on the Si substrate.

The results will contribute to the promotion of technologies with important applications in the field of cyber-mechatronic systems. It is actually an attempt to achieve as many types of submicronic patterns that can be used subsequently to obtain complex mechatronic systems.

\section{References}

[1] I. Verdinas, E. Cretu - "Elements of Nanotechnology", Bucharest, 2007 (in Romanian).

[2] S.E. Lyshevski - "Nano- and MicroElectromechanical Systems: Fundamentals of Nano- and Microengineering", CRC Press; 2nd edition, 2005.

[3] I. Stamatin - "Nanomaterials applications in biosensors, energy sources, medicine, biology. Elements of nanotechnology", university of Bucharest, 2008 (in Romanian).

[4] M. Căldăraru, F. Căldăraru - "Introduction to microelectronics", university course, Cavallioti, 2009.

[5] M. Elwenspoek, R. Wiegerink - „Mechanical Microsensors", Springer-Verlag Berlin Heidelberg, 2001. 
[6] A. Dumitriu - "Basics of mechatronic systems", vol. 1, course, Transilvania University, Brasov, 2006.

[7] M. Nadim, W. Kirt - „An introduction to Microelectromechanical Systems Engineering", Artech House, Inc., 2004.

[8] J.J. Allen - „Microelectromechanical Systems Design", CRC Press, Taylor \& Francis Group, 2005.

[9] S.E. Lyshevski - "MEMS and NEMS - Systems, Devices and Structures", CRC Press; $2^{\text {nd }}$ edition, 2005.
[10] B. Bhushan - "Springer Handbook of Nanotechnology", Springer Science Inc.., 2007.

[11] J.E. Bjorkholm - "EUV Lithography-The

[12] Successor to Optical Lithography?", Intel Technol. J. 2, 1-8, 1998.

[13] W. Menz, J. Mohr, O. Paul - „Microsystem Technology”, Wiley-VCH Verlag GmbH, 2001.

[14] K. Takahata - „Micro Electronic and Mechanical Systems", In-the, Croatia, 2009].

[15] K. Kalantar-zadeh, B. Fry - "NanotechnologyEnabled Sensors", Springer, 2008. 